\title{
Effects of the collagen-glycosaminoglycan mesh on gene expression as determined by using principal component analysis-based unsupervised feature extraction
}

\author{
Y-h. Taguchi ${ }^{1, *(D)}$ and Turki Turki ${ }^{2}$ (D) \\ 1 Department of Physics, Chuo University, Tokyo 112-8551, Japan \\ 2 Department of Computer Science, King Abdulaziz University, Jeddah 21589,Saudi Arabia; \\ tturki@kau.edu.sa \\ * Correspondence:tag@granular.com; Tel.: +81-3-3817-1791
}

Citation: Taguchi, Y-h.; Turki, T.

Effects of the

collagen-glycosaminoglycan mesh on gene expression as determined by using principal component

analysis-based unsupervised feature extraction. Polymers 2021, 1, 0.

https://doi.org/

Received:

Accepted:

Published:

Publisher's Note: MDPI stays neutral with regard to jurisdictional claims in published maps and institutional affiliations.

Copyright: (c) 2021 by the authors. Submitted to Polymers for possible open access publication under the terms and conditions of the Creative Commons Attribution (CC BY) license (https://creativecommons.org/licenses/by/4.0/).

\begin{abstract}
Development of the medical applications for substances or materials that contact the cells is important. Hence, it is necessary to elucidate how substance that surround cells affect the gene expression during incubation. Here, we compared the gene expression profiles of cell lines that were in contact with the collagen-glycosaminoglycan mesh and control cells. Principal component analysis-based unsupervised feature extraction was applied to identify genes with altered expression during incubation in the treated cell lines but not in the controls. The identified genes were enriched in various biological terms. Our method also outperformed a conventional methodology, namely, gene selection based on linear regression with time course.
\end{abstract}

Keywords: Feature Extraction; Tissue Engineering; Microarray Data; Applications in Biology and Medicine

\section{Introduction}

Several factors are known to affect cell division; one such effective factor is the contact with solid materials (or substance) [1]. Regulating the cell division process using biomaterials is the central theme of tissue engineering. The effect of tissue engineering scaffold is especially important because tissue engineering cannot be conducted without equipment that can store cell lines. Collagen-glycosaminoglycan mesh is one such important biomaterial because it is used to aid wound healing [2]. Although Klappericha and Bertozzi [3] once investigated the effect of collagen-glycosaminoglycan mesh on cell division cycles using microarray analysis, the small number of samples studied prevented them from identifying the genes whose expression significantly varied during development and whose expression profiles were distinct between controls and treated cells. Although they selected genes associated with $P$-values less than 0.001 , considering the number of genes as $10^{4}$, it is far below significant.

The recently proposed principal component analysis (PCA)-based unsupervised feature extraction (FE) [4] has the ability to identify genes with expression profiles that were significantly different using small number of samples. In this study, we successfully applied PCA-based unsupervised FE to determine the gene expression profiles during cell division of cells in the control conditions and in contact with collagen-glycosaminoglycan mesh. The identified genes were found to be associated with several enrichment terms with considerable biological significance.

\section{Materials and Methods}

\subsection{Gene expression profiles}

Gene expression profiles were downloaded from the Gene Expression Omnibus (GEO) database (GEO ID: GSE6432). The dataset in GSE6432_series_matrix.txt.gz is 
Table 1. The number of samples with the gene expression profiles. Treated means contact with the collagen-glycosaminoglycan mesh.

\begin{tabular}{ccccccccc}
\hline Conditions & $\mathbf{1} \mathbf{h r}$ & $\mathbf{2} \mathbf{h r s}$ & $\mathbf{4} \mathbf{h r s}$ & $\mathbf{8} \mathbf{h r s}$ & $\mathbf{1 2} \mathbf{h r s}$ & $\mathbf{2 4} \mathbf{h r s}$ & $\mathbf{4 8} \mathbf{h r s}$ & total \\
\hline treated & 3 & 2 & 3 & 4 & 3 & 2 & 2 & 10 \\
control & 1 & 2 & 3 & 2 & 3 & 1 & 1 & 13
\end{tabular}

available in the Series Matrix File(s) section. It consists of 32 gene expression profiles of the IMR90 cell lines, and the relevant details are provided in Table 1.

\subsection{PCA-based unsupervised FE}

Gene expression profiles are formatted as matrices $x_{i j} \in \mathbb{R}^{22283 \times 19}$ for treated cells and $x_{i j} \in \mathbb{R}^{22283 \times 13}$ for control cells, where $x_{i j}$ denotes gene expression of the $i$ th probe at the $j$ th sample. Before applying singular value decomposition (SVD), they were standardized as

$$
\begin{aligned}
& \sum_{i} x_{i j}=0 \\
& \sum_{i} x_{i j}^{2}=22283
\end{aligned}
$$

After applying SVD, we got the left hand singular value vector $u_{\ell i}$, which corresponded to the principal component score attributed to the probes, and right hand singular value vector $v_{\ell j}$, which corresponded to the principal component loadings attributed to the samples, if we interpreted the application of SVD as PCA.

In order to see which $v_{\ell j}$ is coincident with time points, we applied linear regression as:

$$
v_{\ell j}=a_{\ell}+b_{\ell} t_{j}
$$

where $a_{\ell}$ and $b_{\ell}$ are regression coefficients and $t_{j}$ is the time point (hrs in Table 1) associated with the $j$ th sample. We used the $\mathrm{Im}$ function in R [5], and the obtained $P$-values were corrected using the Benjamini-Hochberg criterion [4]. $v_{3 j}$ for treated cell is associated with the adjusted $P$-values less than 0.05 , whereas $v_{\ell j} \mathrm{~s}$ for control cell is not associated with adjusted $P$-values less than 0.05 . This result is appropriate because the simple cell division process may not be associated with any time development other than cell senescence [6], which might not be detected in only 24 hours.

Probes are selected by assuming that $u_{3 i}$, associated with $v_{3 j}$, obeys the Gaussian distribution (null hypothesis) by assigning $P$-values to probes as:

$$
P_{i}=P_{\chi^{2}}\left[>\left(\frac{u_{3 i}}{\sigma_{3}}\right)^{2}\right]
$$

, where $P_{\chi^{2}}[>x]$ is the cumulative $\chi^{2}$ distribution, the argument is larger than $x$, and $\sigma_{3}$ is the standard deviation. Thus, 324 probes associated with the adjusted $P$-value less than 0.01 were selected for the treated cell lines.

\subsection{Gene selection using linear regression}

As an alternative method to PCA-based unsupervised FE, we utilized linear regressionbased FE. Linear regression is applied to $x_{i j}$ as

$$
x_{i j}=a_{i}+b_{i} t_{j}
$$

where $a_{i}$ and $b_{i}$ are regression coefficients and $t_{j}$ is the time point (hrs in Table 1) associated with the $j$ th sample. Subsequently, is associated with adjusted $P$-values less than

0.01 were selected. The number of probes selected for treated cell lines was 813 , and no probes were selected for the control cell lines. 
Table 2. The top 10 enriched terms in "GO Biological Process 2021" using Enrichr. Overlap is the number of genes common between the genes uploaded and the genes in the category divided by the number of genes in the category. Probes, whose associated genes were uploaded to Enrichr, were identified using PCA-based unsupervised FE.

\begin{tabular}{|c|c|c|c|}
\hline Term & Overlap & P-value & $\begin{array}{l}\text { Adjusted } \\
\text { P-value }\end{array}$ \\
\hline regulation of apoptotic process (GO:0042981) & $41 / 742$ & $1.18 \times 10^{-16}$ & $2.52 \times 10^{-13}$ \\
\hline $\begin{array}{l}\text { SRP-dependent cotranslational protein targeting to } \\
\text { membrane (GO:0006614) }\end{array}$ & $17 / 90$ & $4.06 \times 10^{-16}$ & $4.33 \times 10^{-13}$ \\
\hline $\begin{array}{l}\text { cotranslational protein targeting to membrane } \\
\text { (GO:0006613) }\end{array}$ & $17 / 94$ & $8.78 \times 10^{-16}$ & $6.24 \times 10^{-13}$ \\
\hline protein targeting to ER (GO:0045047) & $17 / 103$ & $4.36 \times 10^{-15}$ & $2.33 \times 10^{-12}$ \\
\hline cytoplasmic translation (GO:0002181) & $16 / 93$ & $1.44 \times 10^{-14}$ & $6.15 \times 10^{-12}$ \\
\hline $\begin{array}{l}\text { nuclear-transcribed mRNA catabolic process, } \\
\text { nonsense-mediated decay (GO:0000184) }\end{array}$ & $17 / 113$ & $2.16 \times 10^{-14}$ & $7.70 \times 10^{-12}$ \\
\hline cellular protein metabolic process (GO:0044267) & $27 / 417$ & $7.60 \times 10^{-13}$ & $2.10 \times 10^{-10}$ \\
\hline peptide biosynthetic process (GO:0043043) & $18 / 162$ & $7.89 \times 10^{-13}$ & $2.10 \times 10^{-10}$ \\
\hline $\begin{array}{l}\text { negative regulation of programmed cell death } \\
\text { (GO:0043069) }\end{array}$ & $25 / 381$ & $4.24 \times 10^{-12}$ & $1.00 \times 10^{-9}$ \\
\hline $\begin{array}{l}\text { nuclear-transcribed mRNA catabolic process } \\
\text { (GO:0000956) }\end{array}$ & $17 / 171$ & $2.11 \times 10^{-11}$ & $4.51 \times 10^{-9}$ \\
\hline
\end{tabular}

The top ranked term in GO biological process (BP) (Table 2) is "regulation of apoptotic process". Na et al reported [9] that collagen-glycosaminoglycan has an antiapoptosis effect. Thus, the fact that this term is ranked first is reasonable. 
Table 3. The top 10 enriched terms in "GO Cellular Component 2021" using Enrichr. Overlap is the number of genes common between the genes uploaded and the genes in the category divided by the number of genes in the category. Probes, whose associated genes were uploaded to Enrichr, were identified using PCA-based unsupervised FE.

\begin{tabular}{|c|c|c|c|}
\hline Term & Overlap & P-value & $\begin{array}{c}\text { Adjusted } \\
\text { P-value }\end{array}$ \\
\hline focal adhesion (GO:0005925) & $43 / 387$ & $2.22 \times 10^{-29}$ & $4.77 \times 10^{-27}$ \\
\hline cell-substrate junction (GO:0030055) & $43 / 3$ & $4.69 \times 10^{-29}$ & $5.04 \times 10^{-27}$ \\
\hline intracellular organelle lumen (GO:0070013) & $40 / 848$ & $5.63 \times 10^{-14}$ & $4.03 \times 10^{-12}$ \\
\hline $\begin{array}{l}\text { collagen-containing } \quad \text { extracellular matrix } \\
\text { (GO:0062023) }\end{array}$ & $25 / 380$ & $4.00 \times 10^{-12}$ & $2.15 \times 10^{-10}$ \\
\hline endoplasmic reticulum lumen (GO:0005788) & $21 / 285$ & $2.78 \times 10^{-11}$ & $1.19 \times 10^{-9}$ \\
\hline cytosolic large ribosomal subunit (GO:0022625) & $10 / 55$ & $7.95 \times 10^{-10}$ & $2.85 \times 10^{-8}$ \\
\hline large ribosomal subunit (GO:0015934) & $10 / 59$ & $1.64 \times 10^{-9}$ & $5.03 \times 10^{-8}$ \\
\hline ribosome (GO:0005840) & $10 / 62$ & $2.72 \times 10^{-9}$ & $7.30 \times 10^{-8}$ \\
\hline secretory granule lumen (GO:0034774) & $19 / 316$ & $7.48 \times 10^{-9}$ & $1.79 \times 10^{-7}$ \\
\hline ficolin-1-rich granule lumen (GO:1904813) & $12 / 123$ & $2.50 \times 10^{-8}$ & $5.37 \times 10^{-7}$ \\
\hline
\end{tabular}

Table 4. The top 10 enriched terms in "KEGG 2021 Human" using Enrichr. Overlap is the number of genes common between the genes uploaded and the genes in the category divided by the number of genes in the category. Probes, whose associated genes were uploaded to Enrichr, were identified using PCA-based unsupervised FE.

\begin{tabular}{lccc}
\hline Term & Overlap & P-value & $\begin{array}{c}\text { Adjusted } \\
\text { P-value }\end{array}$ \\
\hline Coronavirus disease & $21 / 232$ & $5.36 \times 10^{-13}$ & $1.22 \times 10^{-10}$ \\
Ribosome & $17 / 158$ & $5.88 \times 10^{-12}$ & $6.67 \times 10^{-10}$ \\
Legionellosis & $9 / 57$ & $2.09 \times 10^{-8}$ & $1.58 \times 10^{-6}$ \\
Salmonella infection & $16 / 249$ & $4.75 \times 10^{-8}$ & $2.70 \times 10^{-6}$ \\
IL-17 signaling pathway & $10 / 94$ & $1.64 \times 10^{-7}$ & $7.46 \times 10^{-6}$ \\
Glycolysis / Gluconeogenesis & $8 / 67$ & $1.20 \times 10^{-6}$ & $4.52 \times 10^{-5}$ \\
Lipid and atherosclerosis & $13 / 215$ & $1.75 \times 10^{-6}$ & $5.69 \times 10^{-5}$ \\
Protein digestion and absorption & $9 / 103$ & $3.65 \times 10^{-6}$ & $1.04 \times 10^{-4}$ \\
Focal adhesion & $12 / 201$ & $5.03 \times 10^{-6}$ & $1.17 \times 10^{-4}$ \\
PI3K-Akt signaling pathway & $16 / 354$ & $5.14 \times 10^{-6}$ & $1.17 \times 10^{-4}$ \\
\hline
\end{tabular}

Other than these three categories, there are some additional categories that support 82 the suitability of our analysis. For example, "ARCHS4 Cell-lines" lists the IMR90, which 83 is the cell line used in this study, as the top ranked cell line (Table 5). 
Table 5. The top 10 enriched terms in "ARCHS4 Cell-lines" using Enrichr. Overlap is the number of genes common between the genes uploaded and the genes in the category divided by the number of genes in the category. Probes, whose associated genes were uploaded to Enrichr, were identified using PCA-based unsupervised FE.

\begin{tabular}{lccc}
\hline Term & Overlap & P-value & $\begin{array}{c}\text { Adjusted } \\
\text { P-value }\end{array}$ \\
\hline IMR90 & $89 / 2395$ & $1.56 \times 10^{-24}$ & $1.95 \times 10^{-22}$ \\
NHDF & $79 / 2395$ & $2.70 \times 10^{-18}$ & $1.69 \times 10^{-16}$ \\
BJ CELL & $72 / 2395$ & $2.02 \times 10^{-14}$ & $8.41 \times 10^{-1} 3$ \\
HUVEC & $64 / 2395$ & $1.61 \times 10^{-10}$ & $5.04 \times 10^{-9}$ \\
T24 & $62 / 2395$ & $1.23 \times 10^{-9}$ & $3.09 \times 10^{-8}$ \\
T98G & $59 / 2395$ & $2.22 \times 10^{-8}$ & $4.63 \times 10^{-7}$ \\
BT549 & $56 / 2395$ & $3.28 \times 10^{-7}$ & $5.12 \times 10^{-6}$ \\
DU145 & $56 / 2395$ & $3.28 \times 10^{-7}$ & $5.12 \times 10^{-6}$ \\
CAKI1 & $55 / 2395$ & $7.68 \times 10^{-7}$ & $9.60 \times 10^{-6}$ \\
U87 & $55 / 2395$ & $7.68 \times 10^{-7}$ & $9.60 \times 10^{-6}$ \\
\hline
\end{tabular}

Moreover, although it is not the top ranked term, "FETAL LUNG", from which IMR90 cell lines were derived, is ranked within the top 10 ranked terms in "ARCHS4 Tissues" (Table 6).

Table 6. The top 10 enriched terms in "ARCHS4 Tissues" using Enrichr. Overlap is the number of genes common between the genes uploaded and the genes in the category divided by the number of genes in the category. Probes, whose associated genes were uploaded to Enrichr, were identified using PCA-based unsupervised FE.

\begin{tabular}{lccc}
\hline Term & Overlap & P-value & $\begin{array}{c}\text { Adjusted } \\
\text { P-value }\end{array}$ \\
\hline MYOBLAST & $87 / 2316$ & $3.16 \times 10^{-24}$ & $3.38 \times 10^{-22}$ \\
FIBROBLAST & $86 / 2316$ & $1.45 \times 10^{-23}$ & $7.76 \times 10^{-22}$ \\
FORESKIN FIBROBLAST & $72 / 2316$ & $3.58 \times 10^{-15}$ & $1.28 \times 10^{-13}$ \\
BLOOD DENDRITIC CELLS & $66 / 2316$ & $4.31 \times 10^{-12}$ & $1.15 \times 10^{-10}$ \\
DENDRITIC CELL & $63 / 2316$ & $1.13 \times 10^{-10}$ & $2.02 \times 10^{-9}$ \\
OSTEOBLAST & $63 / 2316$ & $1.13 \times 10^{-10}$ & $2.02 \times 10^{-9}$ \\
KUPFFER CELL & $62 / 2316$ & $3.22 \times 10^{-10}$ & $4.30 \times 10^{-9}$ \\
VASCULAR SMOOTH MUSCLE & $62 / 2316$ & $3.22 \times 10^{-10}$ & $4.30 \times 10^{-9}$ \\
FETAL LUNG & $61 / 2316$ & $8.95 \times 10^{-10}$ & $9.58 \times 10^{-9}$ \\
LIVER (BULK TISSUE) & $61 / 2316$ & $8.95 \times 10^{-10}$ & $9.58 \times 10^{-9}$ \\
\hline
\end{tabular}

Although we have provided only a few examples, our results suggest that our analysis was robust.

\section{Discussion}

Although we have successfully applied our methodology to the data set, one might wonder whether more conventional method can achieve similar performance. Since this dataset was generated using archaic technology, namely, microarray, more modernized methodologies adapted to high-throughput sequencing technology (e.g., edgeR [11] or DESeq2 [12]) cannot be employed. As for the archaic technologies adapted to microarray (e.g., SAM [13] and limma [14]) cannot be employed as well because they can deal with only categorical classification, whereas we need to identify genes whose expression is altered as a numerical variable (hours). Thus, we decided to employ more a more conventional methodology than SAM or limma, namely, gene selection using linear regression. 
Table 7. The top 10 enriched terms in "GO Biological Process 2021" using Enrichr. Overlap is the number of genes common between the genes uploaded and the genes in the category divided by the number of genes in the category. Probes, whose associated genes were uploaded to Enrichr, were identified using linear regression. linear regression-based FE and uploaded the gene symbols associated with the identified probes to Enrichr. When considering only the number of probes selected, it was better than the PCA-based unsupervised FE, which could identify only 324 probes. Selecting no probes for the control cell lines is the same as PCA-based unsupervised FE. Thus, it seems that the application of PCA-based unsupervised FE, instead of linear regression, was not productive.

Nevertheless, if we consider the performance of the enrichment analysis more carefully, this impression is reversed. Full list of probes, genes, and the results of enrichment analysis are provided in the supplementary material (Data S2). First, for "GO BP 2021", in which PCA-based unsupervised FE ranked apoptosis first (Tables 2, and 7), although the top ranked term "regulation of apoptotic process" in Table 2 is associated with adjusted $P$-value as small as $2.52 \times 10^{-13}$, the top ranked term in Table 7 is associated with adjusted $P$-value as large as $4.56 \times 10^{-2}$, which is much less significant. Even the tenth ranked term in Table 2 is more significant than the top ranked term in Table 7. Generally, more genes uploaded have more opportunity to be associated with more significant enrichment. Nevertheless, genes associated with 813 probes, which is greater than the 324 probes identified using PCA-based unsupervised FE, could be associated with the less significant terms. This definitely suggests the inferiority of linear regression as compared to PCA-based unsupervised FE.
As described in the Materials and Methods section, we identified 813 probes using

\begin{tabular}{|c|c|c|c|}
\hline Term & Overlap & P-value & $\begin{array}{l}\text { Adjusted } \\
\text { P-value }\end{array}$ \\
\hline $\begin{array}{l}\text { actin polymerization or depolymerization } \\
\text { (GO:0008154) }\end{array}$ & $9 / 50$ & $4.05 \times 10^{-5}$ & $4.56 \times 10^{-2}$ \\
\hline $\begin{array}{l}\text { rRNA-containing ribonucleoprotein complex export } \\
\text { from nucleus (GO:0071428) }\end{array}$ & $4 / 7$ & $4.22 \times 10^{-5}$ & $4.56 \times 10^{-2}$ \\
\hline $\begin{array}{l}\text { positive regulation of protein modification process } \\
\text { (GO:0031401) }\end{array}$ & $20 / 214$ & $4.32 \times 10^{-5}$ & $4.56 \times 10^{-2}$ \\
\hline $\begin{array}{l}\text { transmembrane receptor protein tyrosine kinase sig- } \\
\text { naling pathway (GO:0007169) }\end{array}$ & $30 / 404$ & $5.44 \times 10^{-5}$ & $4.56 \times 10^{-2}$ \\
\hline protein stabilization (GO:0050821) & $17 / 179$ & $1.31 \times 10^{-4}$ & $7.08 \times 10^{-2}$ \\
\hline regulation of lipid biosynthetic process (GO:0046890) & $7 / 35$ & $1.46 \times 10^{-4}$ & $7.08 \times 10^{-2}$ \\
\hline $\begin{array}{l}\text { regulation of cellular metabolic process } \\
(\mathrm{GO}: 0031323)\end{array}$ & $8 / 47$ & $1.63 \times 10^{-4}$ & $7.08 \times 10^{-2}$ \\
\hline $\begin{array}{l}\text { positive regulation of cellular protein metabolic pro- } \\
\text { cess (GO:0032270) }\end{array}$ & $12 / 102$ & $1.74 \times 10^{-4}$ & $7.08 \times 10^{-2}$ \\
\hline regulation of stress fiber assembly (GO:0051492) & $10 / 74$ & $1.90 \times 10^{-4}$ & $7.08 \times 10^{-2}$ \\
\hline regulation of mRNA catabolic process (GO:0061013) & $13 / 122$ & $2.58 \times 10^{-4}$ & $7.65 \times 10^{-2}$ \\
\hline
\end{tabular}


Table 8. The top 10 enriched terms in "GO Cellular Component 2021" using Enrichr. Overlap is the number of genes common between the genes uploaded and the genes in the category divided by the number of genes in the category. Probes, whose associated genes were uploaded to Enrichr, were identified using linear regression.

\begin{tabular}{|c|c|c|c|}
\hline Term & Overlap & P-value & $\begin{array}{l}\text { Adjusted } \\
\text { P-value }\end{array}$ \\
\hline focal adhesion (GO:0005925) & $39 / 387$ & $1.41 \times 10^{-9}$ & $3.39 \times 10^{-7}$ \\
\hline (GO:0030055) & $39 /$ & $2.34 \times 1$ & $3.39 \times 10^{-7}$ \\
\hline 015629) & $25 / 316$ & $8.27 \times 10$ & $7.97 \times 10^{-3}$ \\
\hline Imen (GO:0070013) & $49 / \varepsilon$ & $2.02 \times 1$ & $1.46 \times 10$ \\
\hline nucles & $186 / 4$ & $1.06 \times 1$ & $5.51 \times 10^{-2}$ \\
\hline $\begin{array}{l}\text { collagen-containing } \quad \text { extracellular } \\
\text { (GO:0062023) }\end{array}$ & $25 / 380$ & $1.30 \times 10^{-3}$ & $5.51 \times 10^{-2}$ \\
\hline cytoplasmic stress granule (GO:0010494) & $8 / 65$ & $1.54 \times 10^{-3}$ & $5.51 \times 10^{-2}$ \\
\hline $\begin{array}{l}\text { intracellular membrane-bounded } \quad \text { organelle } \\
\text { (GO:0043231) }\end{array}$ & $210 / 5192$ & $1.66 \times 10^{-3}$ & $5.51 \times 10^{-2}$ \\
\hline endoplasmic reticulum lumen (GO:0005788) & & $1.81 \times 10^{-3}$ & $5.51 \times 10^{-2}$ \\
\hline $\begin{array}{l}\text { intracellular non-membrane-bounded organelle } \\
\text { (GO:0043232) }\end{array}$ & $58 / 1158$ & $1.91 \times 10^{-3}$ & $5.51 \times 10^{-2}$ \\
\hline
\end{tabular}

Table 9. The top 10 enriched terms in "KEGG 2021 Human" using Enrichr. Overlap is the number of genes common between the genes uploaded and the genes in the category divided by the number of genes in the category. Probes, whose associated genes were uploaded to Enrichr, were identified using linear regression.

\begin{tabular}{lccc}
\hline Term & Overlap & P-value & $\begin{array}{c}\text { Adjusted } \\
\text { P-value }\end{array}$ \\
\hline PI3K-Akt signaling pathway & $28 / 354$ & $3.17 \times 10^{-5}$ & $9.30 \times 10^{-3}$ \\
Sphingolipid signaling pathway & $13 / 119$ & $2.01 \times 10^{-4}$ & $1.66 \times 10^{-2}$ \\
Arrhythmogenic right ventricular cardiomyopathy & $10 / 77$ & $2.65 \times 10^{-4}$ & $1.66 \times 10^{-2}$ \\
Antigen processing and presentation & $10 / 78$ & $2.95 \times 10^{-4}$ & $1.66 \times 10^{-2}$ \\
Hepatitis C & $15 / 157$ & $2.99 \times 10^{-4}$ & $1.66 \times 10^{-2}$ \\
Salmonella infection & $20 / 249$ & $3.39 \times 10^{-4}$ & $1.66 \times 10^{-2}$ \\
Hippo signaling pathway & $15 / 163$ & $4.47 \times 10^{-4}$ & $1.87 \times 10^{-2}$ \\
Tight junction & $15 / 169$ & $6.54 \times 10^{-4}$ & $2.29 \times 10^{-2}$ \\
Protein processing in endoplasmic reticulum & $15 / 171$ & $7.39 \times 10^{-4}$ & $2.29 \times 10^{-2}$ \\
AMPK signaling pathway & $12 / 120$ & $7.81 \times 10^{-4}$ & $2.29 \times 10^{-2}$ \\
\hline
\end{tabular}


Table 10. The top 10 enriched terms in "ARCHS4 Cell-lines" using Enrichr. Overlap is the number of genes common between the genes uploaded and the genes in the category divided by the number of genes in the category. Probes, whose associated genes were uploaded to Enrichr, were identified using linear regression.

\begin{tabular}{lccc}
\hline Term & Overlap & P-value & $\begin{array}{c}\text { Adjusted } \\
\text { P-value }\end{array}$ \\
\hline IMR90 & $128 / 2395$ & $8.49 \times 10^{-8}$ & $1.06 \times 10^{-5}$ \\
HUVEC & $113 / 2395$ & $1.54 \times 10^{-4}$ & $9.65 \times 10^{-3}$ \\
NHDF & $112 / 2395$ & $2.35 \times 10^{-4}$ & $9.78 \times 10^{-3}$ \\
BT549 & $103 / 2395$ & $6.32 \times 10^{-3}$ & $1.62 \times 10^{-1}$ \\
BJ CELL & $101 / 2395$ & $1.17 \times 10^{-2}$ & $1.62 \times 10^{-1}$ \\
HNSCC & $101 / 2395$ & $1.17 \times 10^{-2}$ & $1.62 \times 10^{-1}$ \\
KNS42 & $101 / 2395$ & $1.17 \times 10^{-2}$ & $1.62 \times 10^{-1}$ \\
NHBE & $101 / 2395$ & $1.17 \times 10^{-2}$ & $1.62 \times 10^{-1}$ \\
U87 & $101 / 2395$ & $1.17 \times 10^{-2}$ & $1.62 \times 10^{-1}$ \\
DAOY & $99 / 2395$ & $2.07 \times 10^{-2}$ & $2.35 \times 10^{-1}$ \\
\hline
\end{tabular}

Table 11. The top 10 enriched terms in "ARCHS4 Tissues" using Enrichr. Overlap is the number of genes common between the genes uploaded and the genes in the category divided by the number of genes in the category. Probes, whose associated genes were uploaded to Enrichr, were identified using linear regression.

\begin{tabular}{lccc}
\hline Term & Overlap & P-value & $\begin{array}{c}\text { Adjusted } \\
\text { P-value }\end{array}$ \\
\hline FIBROBLAST & $116 / 2316$ & $9.27 \times 10^{-6}$ & $5.01 \times 10^{-4}$ \\
VENTRICLE & $116 / 2316$ & $9.27 \times 10^{-6}$ & $5.01 \times 10^{-4}$ \\
ADIPOSE (BULK TISSUE) & $113 / 2316$ & $3.89 \times 10^{-5}$ & $1.05 \times 10^{-3}$ \\
FETAL LUNG & $113 / 2316$ & $3.89 \times 10^{-5}$ & $1.05 \times 10^{-3}$ \\
RESPIRATORY SMOOTH MUSCLE & $112 / 2316$ & $6.14 \times 10^{-5}$ & $1.33 \times 10^{-3}$ \\
OMENTUM & $111 / 2316$ & $9.58 \times 10^{-5}$ & $1.73 \times 10^{-3}$ \\
SUBCUTANEOUS ADIPOSE TISSUE & $106 / 2316$ & $7.60 \times 10^{-4}$ & $1.17 \times 10^{-2}$ \\
MYOBLAST & $104 / 2316$ & $1.61 \times 10^{-3}$ & $2.18 \times 10^{-2}$ \\
NEURONAL EPITHELIUM & $103 / 2316$ & $2.31 \times 10^{-3}$ & $2.77 \times 10^{-2}$ \\
ASTROCYTE & $99 / 2316$ & $8.75 \times 10^{-3}$ & $8.54 \times 10^{-2}$ \\
\hline
\end{tabular}

Although "FETAL LUNG" is fourth ranked in Table 11, adjusted $P$-value is $1.05 \times$ $14810^{-3}$, which is much less significant than that in Table $6\left(9.58 \times 10^{-9}\right)$. Thus, overall, 


\section{Conclusions}

Here, we applied PCA-based unsupervised FE to gene expression profiles for the IMR90 cell lines incubated in collagen-glycosaminoglycan mesh. Whereas no genes whose expression vary as time goes are detected in control cell lines, the expression profiles of several genes were altered during the cell division process. These genes are associated with several enriched biological terms. One conventional method, linear regression was employed for comparison. Although it could select several hundred genes whose expression vary as time passes, their enrichment was inferior to that seen using PCA-based unsupervised FE. Thus, PCA-based unsupervised FE can not only achieve good performance but also outperform a conventional method.

Author Contributions: YHT planned the research, performed analyses. YHT and TT have evaluated the results, discussions, outcomes and wrote and reviewed the manuscript.

Funding: This work was supported by KAKENHI [grant numbers 19H05270, 20H04848, and 20K12067] to YHT.

Data Availability Statement: Data used in this study is available in GEO ID GSE6432.

Conflicts of Interest: The authors declare no conflict of interest. The founders had no role in the design of the study; in the collection, analyses, or interpretation of data; in the writing of the manuscript, or in the decision to publish the results.

\section{References}

1. McMurray, R.J.; Dalby, M.J.; Tsimbouri, P.M. Using biomaterials to study stem cell mechanotransduction, growth and differentiation. Journal of Tissue Engineering and Regenerative Medicine 2015, 9, 528-539, [https:/ / onlinelibrary.wiley.com/doi/pdf/10.1002/term.1957]. doi:https://doi.org/10.1002/term.1957.

2. Ansari, M.; Kordestani, S.S.; Nazralizadeh, S.; Eslami, H. Biodegradable Cell-Seeded Collagen Based Polymer Scaffolds for Wound Healing and Skin Reconstruction. Journal of Macromolecular Science, Part B 2018, 57, 100-109, [https:/ / doi.org/10.1080/00222348.2018.143561 doi:10.1080/00222348.2018.1435617.

3. Klapperich, C.M.; Bertozzi, C.R. Global gene expression of cells attached to a tissue engineering scaffold. Biomaterials 2004, 25, 5631-5641. doi:https://doi.org/10.1016/j.biomaterials.2004.01.025.

4. h. Taguchi, Y. Unsupervised Feature Extraction Applied to Bioinformatics; Springer International Publishing, 2020. doi:10.1007/978-3030-22456-1.

5. R Core Team. R: A Language and Environment for Statistical Computing. R Foundation for Statistical Computing, Vienna, Austria, 2020.

6. Micco, R.D.; Krizhanovsky, V.; Baker, D.; d'Adda di Fagagna, F. Cellular senescence in ageing: from mechanisms to therapeutic opportunities. Nature Reviews Molecular Cell Biology 2020, 22, 75-95. doi:10.1038/s41580-020-00314-w.

7. Huang, D.W.; Sherman, B.T.; Lempicki, R.A. Systematic and integrative analysis of large gene lists using DAVID bioinformatics resources. Nature Protocols 2008, 4, 44-57. doi:10.1038/nprot.2008.211.

8. Kuleshov, M.V.; Jones, M.R.; Rouillard, A.D.; Fernandez, N.F.; Duan, Q.; Wang, Z.; Koplev, S.; Jenkins, S.L.; Jagodnik, K.M.; Lachmann, A.; McDermott, M.G.; Monteiro, C.D.; Gundersen, G.W.; Ma'ayan, A. Enrichr: a comprehensive gene set enrichment analysis web server 2016 update. Nucleic Acids Research 2016, 44, W90-W97, [https:/ /academic.oup.com/nar/articlepdf/44/W1/W90/18788036/gkw377.pdf]. doi:10.1093/nar/gkw377.

9. Na, J.; Bak, D.; Im, S.I.; Choi, H.; Hwang, J.H.; Kong, S.Y.; No, Y.A.; Lee, Y.; Kim, B.J. Anti-apoptotic effects of glycosaminoglycans via inhibition of ERK/AP-1 signaling in TNF- $\alpha$-stimulated human dermal fibroblasts. International Journal of Molecular Medicine 2018. doi:10.3892/ijmm.2018.3483.

10. Murphy, C.M.; Matsiko, A.; Haugh, M.G.; Gleeson, J.P.; O’Brien, F.J. Mesenchymal stem cell fate is regulated by the composition and mechanical properties of collagen-glycosaminoglycan scaffolds. Journal of the Mechanical Behavior of Biomedical Materials 2012, 11, 53-62. Special Issue on Tissue Engineering, doi:https://doi.org/10.1016/j.jmbbm.2011.11.009.

11. McCarthy, D.J.; Chen, Y.; Smyth, G.K. Differential expression analysis of multifactor RNA-Seq experiments with respect to biological variation. Nucleic Acids Research 2012, 40, 4288-4297, [https://academic.oup.com/nar/articlepdf/40/10/4288/25335174/gks042.pdf]. doi:10.1093/nar/gks042.

12. Love, M.I.; Huber, W.; Anders, S. Moderated estimation of fold change and dispersion for RNA-seq data with DESeq2. Genome Biology 2014, 15. doi:10.1186/s13059-014-0550-8.

13. Tusher, V.G.; Tibshirani, R.; Chu, G. Significance analysis of microarrays applied to the ionizing radiation response. Proceedings of the National Academy of Sciences 2001, 98, 5116-5121. doi:10.1073/pnas.091062498.

14. Ritchie, M.E.; Phipson, B.; Wu, D.; Hu, Y.; Law, C.W.; Shi, W.; Smyth, G.K. limma powers differential expression analyses for RNA-sequencing and microarray studies. Nucleic Acids Research 2015, 43, e47. doi:10.1093/nar/gkv007. 